\title{
805 CLONAL, ACTIVATED CD8+ T CELLS RECOGNIZING CARDIAC ALPHA-MYOSIN DRIVE IMMUNE CHECKPOINT INHIBITOR ASSOCIATED MYOCARDITIS IN MICE
}

${ }^{1}$ Margaret Axelrod*, ${ }^{1}$ Wouter Meijers, ${ }^{1} E l i e$ Tannous, ${ }^{1}$ Xiaopeng Sun, ${ }^{1} J u a n$ Qin, ${ }^{1}$ Ayaka Sugiura, ${ }^{1}$ Elizabeth Wescott, ${ }^{1}$ Elles Screever, ${ }^{2}$ Spencer Wei, ${ }^{1}$ Susan Opalenik,

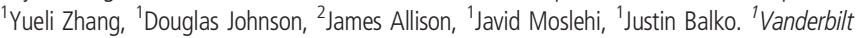
University, Nashville, TN, United States; ${ }^{2}$ MD Anderson Cancer Center, Hayward, CA, United States

Background Nearly half of all U.S. oncology patients meet FDA eligibility criteria to receive treatment with an immune checkpoint inhibitor (ICI). With increasing use of ICIs, preventing, diagnosing and treating immune-related adverse events (irAEs) are urgent clinical challenges. Myocarditis is an uncommon irAE, affecting $<1 \%$ of ICI-treated patients, but is highly fatal, with a mortality rate of nearly $50 \%$. Genetically altered Pdcd1-/-Ctla4 \pm mice die prematurely and specifically due to myocarditis. This model recapitulates the clinical and pathological features of ICI-myocarditis, including abundant cardiac infiltrating CD8 $+\mathrm{T}$ cells. The potential autoantigen(s) involved in ICI-myocarditis are unknown for both human disease and our murine model.

Methods We used Pdcd1-/-Ctla4 \pm mice on the C57BL6 background as a model of ICI-myocarditis. Single cell RNA and T cell receptor (TCR) sequencing was performed on sorted CD45 + cardiac immune cells from four affected Pdcd1-/-Ctla4 \pm mice compared to six healthy wild type mice. The most three clonal TCRs (TCR-A, B, C), derived from two independent Pdcd1-/-Ctla4 \pm mice, were reconstructed using stiTChR and transduced into reporter $\mathrm{T}$ cell lines for antigen discovery. Alpha-myosin was selected as a candidate autoantigen due to lack of presentation in the thymus. Reporter TCR$\mathrm{A}, \mathrm{B}$, and $\mathrm{C}$ cells were screened using a library of overlapping 20 amino acid peptides derived from alpha-myosin in co-culture with bone marrow derived dendritic cells.

Results Treatment with anti-CD8, but not anti-CD4, depleting antibodies rescues survival of Pdcd1-/-Ctla4 \pm mice. Furthermore, adoptive transfer of splenocytes from Pdcd1-/-Ctla4 \pm mice with myocarditis to Rag1-/- recipient mice was sufficient to induce fatal myocarditis. Single cell RNA/TCR sequencing on the cardiac immune infiltrate of Pdcd1-/-Ctla4 \pm mice identified highly activated, clonal CD $8+\mathrm{T}$ cells as the dominant cell population. The TCR-A cell line, the most clonal TCR identified in single cell TCR sequencing, activates NFAT, NFkB, and AP-1 reporters in response to the alpha-myosin epitope VIQYFASI. The TCR-B and TCR-C cell lines activate their reporters in response to the alpha myosin peptide DALLVIQWNIRAFMGVKNWP, indicating that alpha-myosin is an autoantigen in this mouse model of ICI-myocarditis.

Conclusions Clonal, activated CD8 $+\mathrm{T}$ cells are critical for the development of ICI-myocarditis. Alpha-myosin is an autoantigen recognized by the most clonal cardiac CD8 $+\mathrm{T}$ cells. Efforts are currently underway to determine whether human TCRs derived from ICI-myocarditis samples recognize similar antigens. These studies are the first to identify a candidate autoantigen in ICI-myocarditis and may yield new insights into irAE pathogenesis.

Ethics Approval All animal experiments were in accordance with the VUMC Institutional Animal Care and Use Committee (IACUC), protocol \# M2000067

http://dx.doi.org/10.1136/jitc-2021-SITC2021.805 